\title{
BMJ Open Atrial fibrillation ablation with a spring sensor-irrigated contact force-sensing catheter compared with other ablation catheters: systematic literature review and meta-analysis
}

\author{
Laurent Macle, ${ }^{1,2}$ Diana Frame, ${ }^{3}$ Larry M Gache, ${ }^{3}$ George Monir, ${ }^{4}$ Scott J Pollak, ${ }^{4}$ \\ Lee Ming Boo ${ }^{5}$
}

To cite: Macle L, Frame D, Gache LM, et al. Atrial fibrillation ablation with a spring sensor-irrigated contact forcesensing catheter compared with other ablation catheters: systematic literature review and meta-analysis. BMJ Open 2019;9:e023775. doi:10.1136/ bmjopen-2018-023775

- Prepublication history and additional material for this paper are available online. To view these files, please visit the journal online (http://dx.doi. org/10.1136/bmjopen-2018023775).

Received 24 April 2018 Revised 14 November 2018 Accepted 17 April 2019

Check for updates

(C) Author(s) (or their employer(s)) 2019. Re-use permitted under CC BY-NC. No commercial re-use. See rights and permissions. Published by BMJ.

For numbered affiliations see end of article.

Correspondence to

Dr Laurent Macle;

Imacle@mac.com

\section{ABSTRACT}

Objectives The objective of our review was to systematically assess available evidence on the effectiveness, safety and efficiency of a spring sensorirrigated contact force (CF) catheter (THERMOCOOL SMARTTOUCH Catheter (ST)) for percutaneous ablation of paroxysmal or persistent atrial fibrillation (AF), compared with other ablation catheters, or with the ST with the operator blinded to CF data.

Design Systematic literature review and meta-analysis. Background Emerging evidence suggests improved clinical outcomes of AF ablation using CF-sensing catheters; however, reviews to date have included data from multiple, distinct CF technologies.

Methods We conducted a systematic review and metaanalysis of published studies comparing the use of ST versus other ablation catheters for the treatment of AF. A comprehensive search of electronic and manual sources was conducted. The primary endpoint was freedom from recurrent atrial tachyarrhythmia (AT) at 12 months. Procedural and safety data were also analysed.

Results Thirty-four studies enrolling 5004 patients were eligible. The use of ST was associated with increased odds of freedom from AT at 12 months (71.0\%vs60.8\%; OR $1.454,95 \% \mathrm{Cl} 1.12$ to $1.88, \mathrm{p}=0.004)$ over the comparator group, and the effect size was most evident in paroxysmal AF patients (75.6\%vs64.7\%; OR 1.560, 95\% $\mathrm{Cl} 1.09$ to $2.24, p=0.015)$. Procedure and fluoroscopy times were shorter with ST $(p=0.05$ and $p<0.01$, respectively, vs comparator groups). The reduction in procedure time is estimated at $15.5 \mathrm{~min}$ (9.0\%), and fluoroscopy time $4.8 \mathrm{~min}$ (18.7\%). Complication rates, including cardiac tamponade, did not differ between groups.

Conclusions Compared with the use of other catheters, AF ablation using the CF-sensing ST catheter for AF is associated with improved success rates, shorter procedure and fluoroscopy times and similar safety profile.

\section{INTRODUCTION}

Atrial fibrillation (AF) affects an estimated 33 million individuals worldwide and is a major cause of stroke, heart failure and
Strengths and limitations of this study

- Provides a homogenous evaluation of evidence by assessing the effectiveness, safety and efficiency of contact force (CF)-guided atrial fibrillation (AF) ablation using a specific model of open-irrigated CF catheter.

- Used recommended best practices, including a prospectively defined search strategy, inclusion criteria and a statistical analysis plan.

- Data from both randomised and non-randomised studies were included in the analysis due to the limited availability of randomised evidence in this setting.

- There were limited persistent AF studies reporting the primary endpoint and, therefore, the results in this patient population need to be examined further.

death. Catheter ablation is an established treatment option for symptomatic AF when a rhythm control strategy is desired and anti-arrhythmic drug therapy is ineffective or not tolerated. ${ }^{1}$ Improvements in ablation technologies and techniques to safely create more durable lesions could improve the riskbenefit profile of this procedure. ${ }^{2}$ Recent advances in radiofrequency (RF) catheter design include models with real-time monitoring of catheter-to-tissue contact force (CF). Evidence suggests improved clinical success in paroxysmal AF ablation with stable catheter-tissue contact. ${ }^{3}$

Systematic reviews of $\mathrm{AF}$ ablation using CF catheters ${ }^{45}$ have yielded mixed results, in part due to variations in methodologies and the rapidly evolving evidence base. In addition, all meta-analyses published to date have combined outcomes from different CF-sensing technologies, which have distinct physical properties, instructions for use and 
associated electroanatomical mapping systems and software. ${ }^{67}$ As such, differences between CF-sensing technologies may affect clinical outcome, as suggested by a linear relationship between $\mathrm{CF}$ and 12-month success for a fibre optic CF-sensing technology, ${ }^{7}$ while a non-linear relationship between $\mathrm{CF}$ and 12-month success was noted for a spring sensor CF catheter. ${ }^{3}$ To provide a homogeneous evaluation of evidence, the objective of this systematic literature review and meta-analysis was to assess the effectiveness, safety and efficiency of CF-guided AF ablation using a specific model of open-irrigated CF catheter (THERMOCOOL SMARTTOUCH Catheter (ST), Biosense Webster, Irvine, California, USA), compared with any other ablation catheter or with the ST catheter with the operator blinded to CF data. This CF-sensing technology comprises a small spring connecting the ablation tip electrode to the catheter shaft equipped with a magnetic transmitter and sensors. Published evidence on the ST catheter has not been meta-analysed separately for clinical endpoints (safety and efficacy) and procedure efficiency (procedure time, fluoroscopy use) compared with other ablation strategies.

\section{METHODS}

We performed a systematic review of the research question and since the data extracted from qualified studies of our systematic review were sufficient, we performed a meta-analysis. Both were performed using recommended best practices, including a prospectively defined search strategy, inclusion criteria and statistical analysis plan. ${ }^{8}$
The review was conducted under a prospective protocol, without registration with any external entity.

\section{Data source}

A comprehensive search of clinical literature published through 1 August 2017 was conducted. Our search strategy encompassed several electronic databases as well as manual searches (online supplement 1 ). Search results were not restricted by language, although the majority of journals indexed in the databases searched are published in English, and English language titles and abstracts were used for screening where available. In brief, our search strategy included the National Library of Medicine's PubMed database, the Excerpta Medica (EMBASE®) database from Elsevier B.V., the Cochrane Library CENTRAL register, the ClinicalTrials.gov database and manual reference checks.

\section{Inclusion criteria}

Randomised or non-randomised studies were eligible if they reported comparative data for percutaneous $\mathrm{AF}$ ablation using the ST CF-sensing catheter versus any comparator: standard focal non-CF-sensing RF catheters, ST catheters with operator blinded to CF data, singleshot devices (cryoballoon, multielectrode RF) or another CF-sensing catheter. To exclude studies of insufficient sample size, studies were included only if they enrolled at least 10 adult $\mathrm{AF}$ patients and reported at least one outcome of interest (effectiveness or safety). Meeting abstracts, white papers, editorial/commentary and reviews without primary data were excluded. Studies were

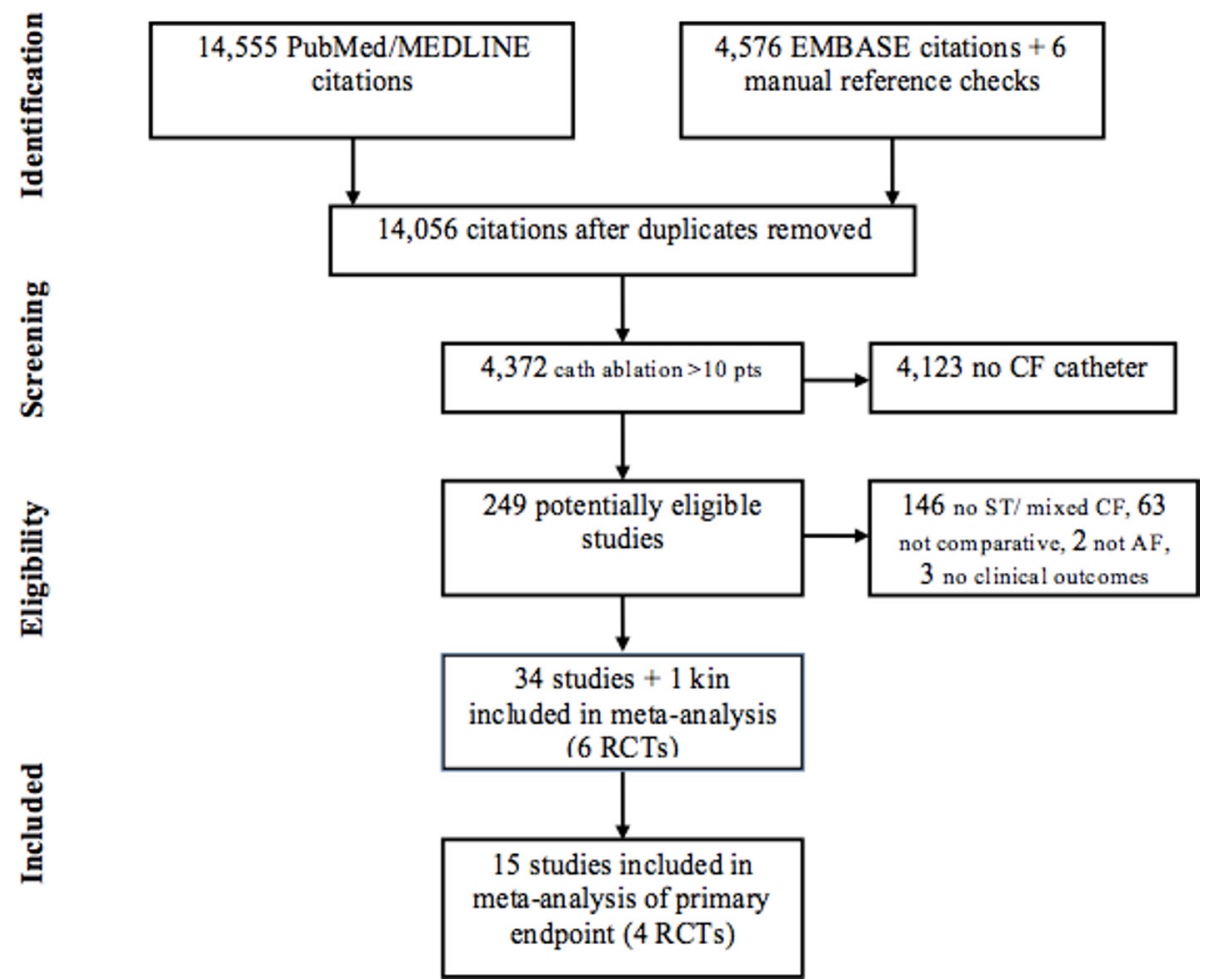

Figure 1 Study attrition. AF, atrial fibrillation; CF, contact force; RCT, randomised controlled trial; ST, THERMOCOOL SMARTTOUCH catheter. 
Table 1 Characteristics of included studies

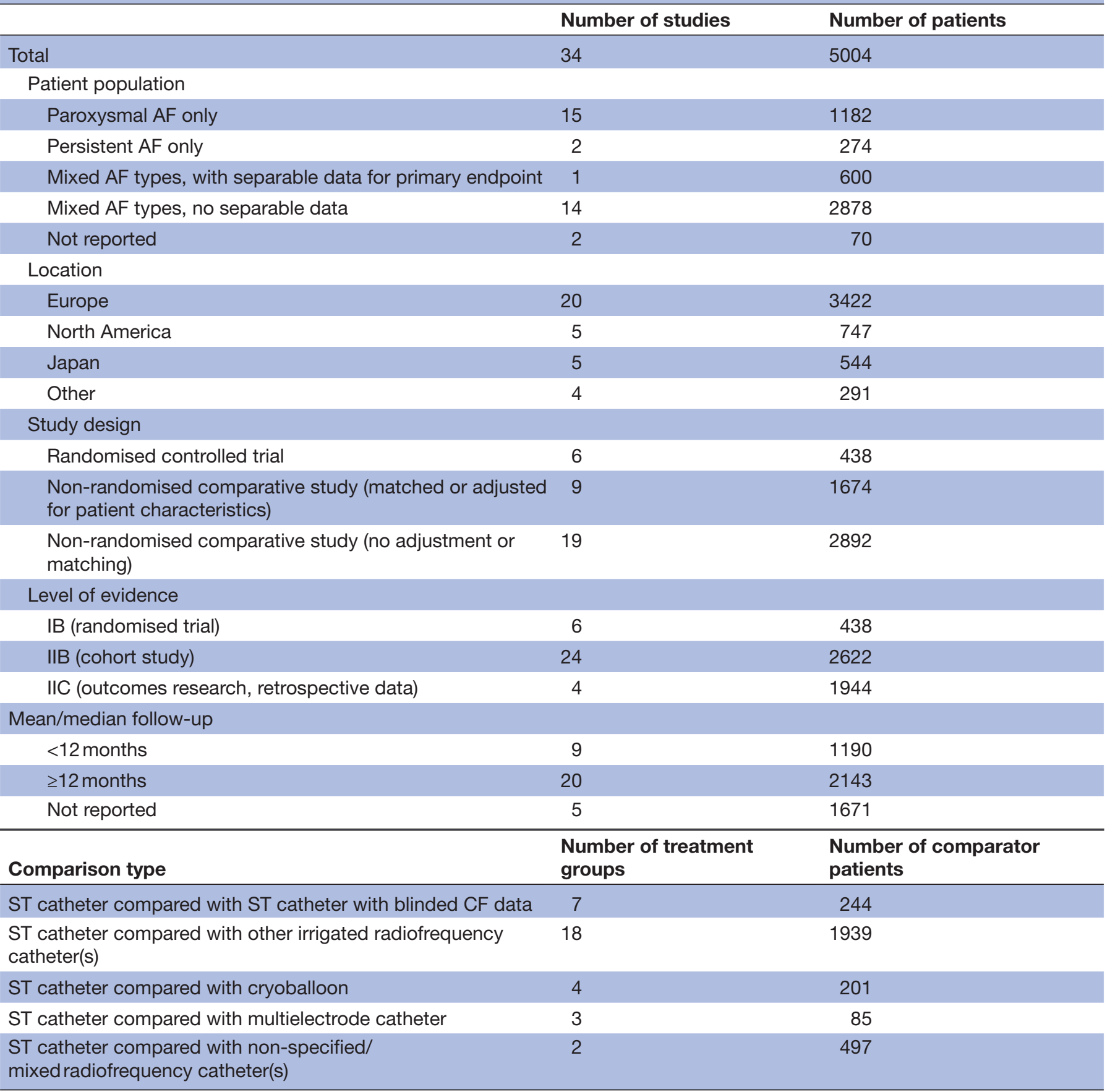

AF, atrial fibrillation; CF, contact force; ST, THERMOCOOL SMARTTOUCH catheter.

not eligible if data could not be separately extracted for the ST catheter.

\section{Statistical analyses}

The primary endpoint was freedom from recurrent atrial tachyarrhythmia (AT) at 12 months. Recurrence of AT was defined as any episode (symptomatic or asymptomatic) of documented atrial arrhythmia lasting $30 \mathrm{~s}$ or more (online supplement 2). Procedural parameters and safety data were also analysed. Sensitivity and subgroup analyses were planned a priori to understand the robustness of the comparisons and to explore potential sources of heterogeneity. Freedom from recurrent AT at 12 months was evaluated using OR; continuous outcomes such as procedure times and fluoroscopy dose were evaluated with Hedges' $g$ differences. Hedges' $g$ is a standardised mean difference, appropriate when outcome definitions, measurement scales or data reporting formats differ among studies. Hedges' $g$ values $<0.2$ indicate a small effect, 0.5 a medium effect, and $>0.8$ a large effect. ${ }^{9}$ Mean differences, using studies which provided means and SD, 
were also computed to provide clinical context for continuous outcomes. The Cochran-Mantel-Haenszel chi test was used to compare the rates of total complications and cardiac tamponade.

We used DerSimonian-Laird (DL) random-effects models for the primary analysis of all effectiveness and efficiency outcomes. We also employed an alternate meta-analytic method, Hartung-Knapp-Sidik-Jonkman (HKSJ), which is increasingly recommended for small and heterogeneous data sets, to assess impact on results. ${ }^{10}$ After fitting the analyses, key patient and study design characteristics, for example, type of AF, comparator and study design, were assessed to explore heterogeneity and examine the robustness of the results, data permitting. Study quality was assessed using the Oxford Center for Evidence-Based Medicine Levels of Evidence. All studies in this review were Levels IB (randomised trial), IIB (individual cohort study) or IIC (outcomes research). The potential for publication bias or small-study effects was assessed using funnel plots and Rucker's Arcsine Test of Asymmetry (online supplement 3). ${ }^{11} 12$ Analyses were performed using Comprehensive Meta-Analysis software (Englewood, New Jersey, USA), V.3 and SAS Software, V.9.2 or higher. Fixed-effects models were not included since heterogeneity was expected among and between the randomised and non-randomised studies.

\section{Patient and public involvement}

Patients were not involved in this study.

\section{RESULTS}

The search identified 34 studies meeting eligibility criteria, including 6 randomised controlled trials. One study was reported in two publications (in English and Hungarian $)^{13}{ }^{14}$; all other studies were published in English. The primary reasons for study exclusion were as follows: no use of the technology of interest (ST), mixed ablation technologies without separable data and non-comparative study design (figure 1). A total of $5004 \mathrm{AF}$ patients were enrolled in the 34 studies (CF: 2038; comparator: 2966). The majority of the patients

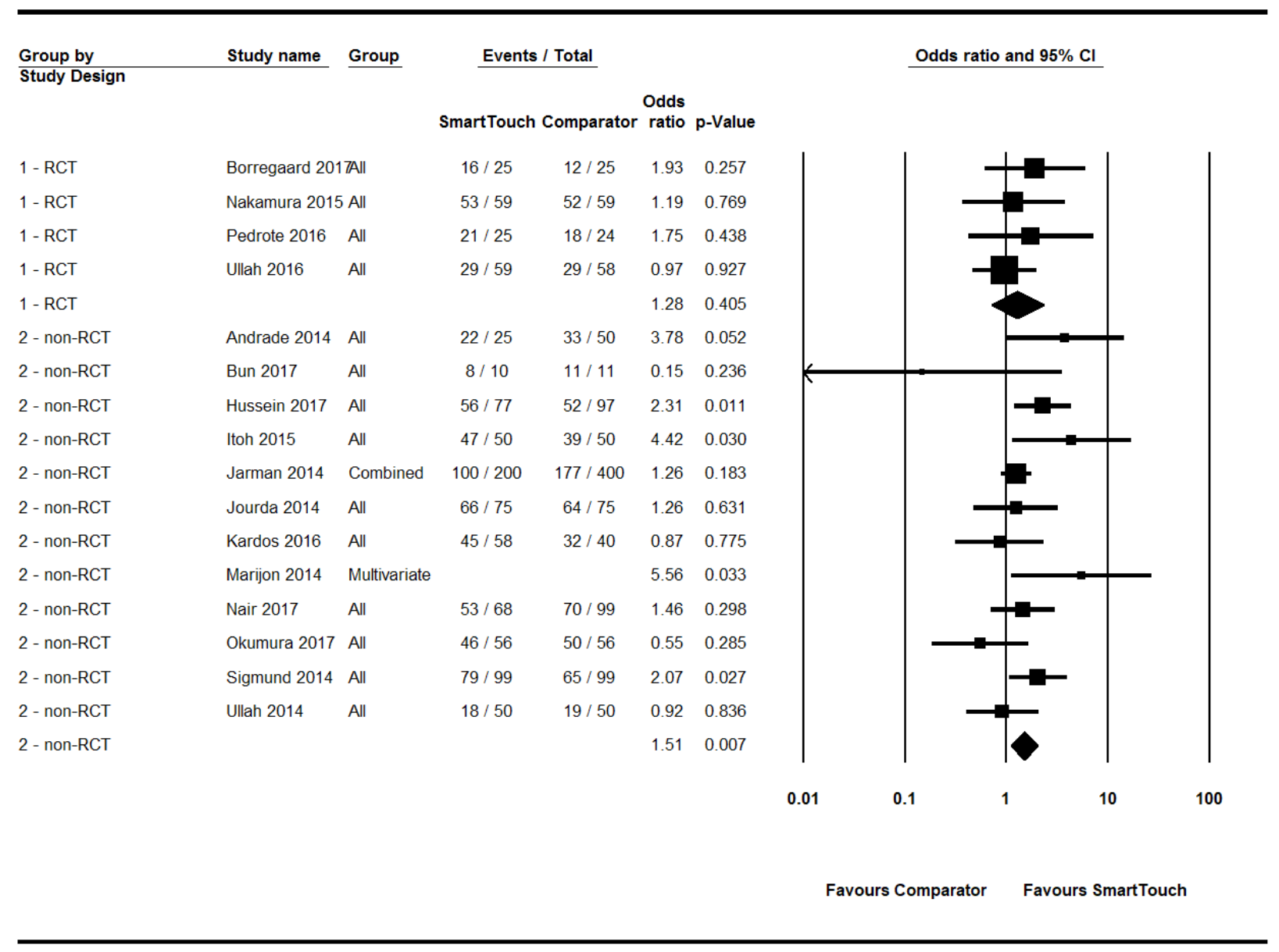

\section{Odds Ratio - Random Effects}

Figure 2 Freedom from AT at 12 months for ST versus comparator ablation catheters, by study design. Total effect - ST versus comparator ablation catheters: $686 / 966$ (71.0\%) versus 744/1223 (60.8\%); OR 1.454, 95\% Cl 1.12 to 1.88, $\mathrm{p}=0.004$; heterogeneity: Cochran's $Q=20.2, d f=15$ ( $p=0.165) ; I^{2}=25.6 \% . R C T: 70.8 \%$ versus $66.9 \%$; OR $1.284,95 \% \mathrm{Cl} 0.71$ to 2.31, $p=0.405$; non-RCT: $71.1 \%$ versus 59.9\%; OR 1.505, 95\% Cl 1.12 to 2.03, $p=0.007$. AT, atrial tachyarrhythmia; RCT, randomised controlled trial; ST, THERMOCOOL SMARTTOUCH catheter. 
Freedom from atrial tachyarrhythmia at 12 months, PAF-only Studies

\begin{tabular}{|c|c|c|c|c|}
\hline \multirow[t]{2}{*}{$\underline{\text { Study name }}$ Group } & \multicolumn{4}{|c|}{ Statistics for each study } \\
\hline & $\begin{array}{l}\text { Odds } \\
\text { ratio }\end{array}$ & $\begin{array}{r}\text { Lower } \\
\text { limit }\end{array}$ & $\begin{array}{l}\text { Upper } \\
\text { limit }\end{array}$ & $\rho$ \\
\hline Andrade 201All & 3.778 & 0.99 & 14.44 & 0.052 \\
\hline Bun $2017 \quad$ All & 0.148 & 0.01 & 3.50 & 0.236 \\
\hline Itoh $2015 \quad$ All & 4.419 & 1.15 & 16.97 & 0.030 \\
\hline Jarman 2014 wo PAF Gro & ps.655 & 1.00 & 2.75 & 0.051 \\
\hline Jourda 2014All & 1.260 & 0.49 & 3.24 & 0.631 \\
\hline Kardos 2016All & 0.865 & 0.32 & 2.33 & 0.775 \\
\hline Marijon 2014Multivariate & 5.556 & 1.15 & 26.93 & 0.033 \\
\hline Nair 2017 All & 1.464 & 0.71 & 3.00 & 0.298 \\
\hline Pedrote 20164ll & 1.750 & 0.43 & 7.19 & 0.438 \\
\hline Ullah 2016 All & 0.967 & 0.47 & 2.00 & 0.927 \\
\hline & 1.560 & 1.09 & 2.24 & 0.015 \\
\hline
\end{tabular}

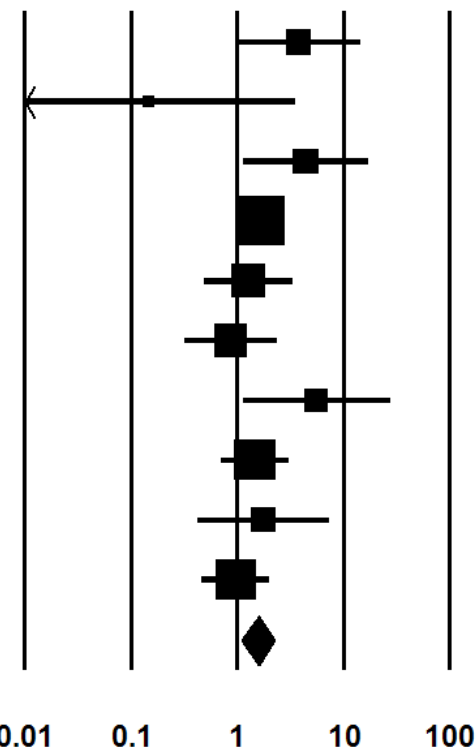

Favours Comparator Favours SmartTouch

Odds Ratio - Random Effects (Note: Multivariate Effect for Marijon 2014)

Figure 3 Freedom from AT at 12 months in paroxysmal AF patients. AF, atrial fibrillation; AT, atrial tachyarrhythmia; PAF, paroxysmal atrial fibrillation; SmartTouch, THERMOCOOL SMARTTOUCH catheter.

had paroxysmal AF (60.3\%). Two-thirds (65.4\%) of all patients in the comparator groups were treated with open-irrigated RF catheters. No studies comparing the ST to other CF-sensing technologies were found. Studies were primarily performed in Europe, with five studies in Japan and five in North America (USA: 3; Canada: 2; USA and Canada: 1) (table 1). Most evidence was Oxford Level of Evidence IIB (individual cohort study).

\section{Effectiveness}

The use of ST was associated with significantly increased odds of the primary endpoint, freedom from AT at 12 months, compared with comparator ablation catheters $(71.0 \%$ vs $60.8 \%$; OR $1.454,95 \%$ CI 1.12 to 1.88 , $\mathrm{p}=0.004$; figure 2). Subgroup analyses demonstrated that the benefit of ST ablation over all other ablation catheters was most evident for paroxysmal AF (75.6\% vs $64.7 \%$; OR $1.560,95 \%$ CI 1.09 to 2.24, $\mathrm{p}=0.015$; figure 3). No difference was found for persistent $\mathrm{AF}$ (51.1\% ST vs $44.9 \%$ comparators; OR $1.287,95 \% \mathrm{CI}$ 0.73 to $2.28, \mathrm{p}=0.385$; figure 4 ), but the sample size was too small (three studies) to draw definitive conclusions. Separating the comparators by type showed no significant difference in freedom from AT at 12 months when the operator was blinded from the CF data using ST catheter or when a single-shot catheter (cryoballoon) was used for ablation (table 2). Freedom from AT at 12 months was significantly higher (OR 1.766, 95\% CI 1.22 to $2.55, \mathrm{p}=0.002)$ in the ST group in comparison to non-CF RF catheters such as irrigated point-by-point RF catheters, remote navigated RF catheter, diamond-tip $\mathrm{RF}$ catheter and unspecified RF catheters. Randomised trials, all with study design comparing $\mathrm{CF}$ visible to $\mathrm{CF}$ blinded groups, showed a smaller, nonsignificant effect compared with non-randomised comparative trials (figure 2). Other patient and study design characteristics including level of evidence, use of matching or adjustment and study sponsorship were not significant sources of variation among studies. Meta-regression was used to investigate possible sources of heterogeneity for freedom from AT at 12 months, and none of the analyses showed statistically significant results. Statistical results were similar when the alternate meta-analytic method, HKSJ, was employed (freedom from AT at 12 months: OR $1.454,95 \%$ CI 1.09 to $1.94, p=0.015$ ). No evidence 
Freedom from Atrial Tachyarrhythmia at 12 months, Persistent-only Studies

\begin{tabular}{|c|c|c|c|c|c|}
\hline \multirow[t]{3}{*}{ Study name } & \multirow[t]{3}{*}{ Group } & \multicolumn{4}{|c|}{ Statistics for each study } \\
\hline & & \multicolumn{4}{|c|}{ Odds Lower Upper } \\
\hline & & ratio & limit & limit & o-Value \\
\hline Hussein 2017 & All & 2.308 & 1.22 & 4.38 & 0.011 \\
\hline Jarman 2014 & Two persAF Groups & 1.000 & 0.63 & 1.60 & 1.000 \\
\hline \multirow[t]{2}{*}{ Ullah 2014} & All & 0.918 & 0.41 & 2.07 & 0.836 \\
\hline & & 1.287 & 0.73 & 2.28 & 0.385 \\
\hline
\end{tabular}

$\begin{array}{lllll}0.01 & 0.1 & 1 & 10 & 100\end{array}$

Odds Ratio - Random Effects

Figure 4 Freedom from AT at 12 months in persistent AF patients. AF, atrial fibrillation; AT, atrial tachyarrhythmia; PersAF, persistent atrial fibrillation; SmartTouch, THERMOCOOL SMARTTOUCH catheter.

of publication bias or small-study effects was found in the funnel plot or Rucker's Arcsine test of asymmetry ( $\mathrm{p}=0.765$; online supplement 3 ).

Fourteen studies also reported acute pulmonary vein (PV) reconnection, which was lower with CF compared with the comparator group (OR $0.448,95 \%$ CI 0.30 to 0.67 , $\mathrm{p} \leq 0.0005)$. When examining the paroxysmal AF subset of patients, acute PV reconnections were significantly lower in the CF group (OR $0.486,95 \%$ CI 0.29 to 0.82 , $\mathrm{p}=0.007$; figure 5). It is important to note that studies

Table 2 Separation of comparators by type of ablation catheter

\begin{tabular}{|c|c|c|c|c|}
\hline & \multicolumn{4}{|l|}{ Comparators } \\
\hline & $\begin{array}{l}\text { All (Estimate }(95 \% \mathrm{Cl}) \text {, } \\
\mathrm{P} \text { value) }\end{array}$ & $\begin{array}{l}\text { Blinding to CF } \\
\text { (Estimate }(95 \% \mathrm{Cl}), \mathrm{P} \\
\text { value) }\end{array}$ & $\begin{array}{l}\text { Single-shot catheter } \\
\text { (Estimate }(95 \% \mathrm{Cl}), \mathrm{P} \\
\text { value) }\end{array}$ & $\begin{array}{l}\text { Non-CF catheter } \\
\text { (Estimate }(95 \% \mathrm{CI}), \mathrm{P} \\
\text { value) }\end{array}$ \\
\hline Freedom from AT (OR) & $\begin{array}{l}1.454 \text { (1.12 to } 1.88) \\
0.004\end{array}$ & $\begin{array}{l}1.243 \text { (0.75 to } 2.06) \\
0.397\end{array}$ & $\begin{array}{l}0.877 \text { (0.49 to } 1.57) \\
0.658^{*}\end{array}$ & $\begin{array}{l}1.766 \text { (1.22 to } 2.55) \\
0.002\end{array}$ \\
\hline $\begin{array}{l}\text { Procedure time } \\
\text { (Hedges' g) }\end{array}$ & $\begin{array}{l}-0.254(-0.50 \text { to }-0.01) \\
0.046\end{array}$ & $\begin{array}{l}-0.414(-0.73 \text { to }-0.10) \\
0.010\end{array}$ & $\begin{array}{l}0.511(-0.62 \text { to } 1.64), \\
0.375\end{array}$ & $\begin{array}{l}-0.440(-0.70 \text { to }-0.18) \\
0.001\end{array}$ \\
\hline $\begin{array}{l}\text { Fluoroscopy time } \\
\text { (Hedges' g) }\end{array}$ & $\begin{array}{l}-0.442(-0.66 \text { to }-0.22) \\
<0.0005\end{array}$ & $\begin{array}{l}-0.248(-0.48 \text { to }-0.01) \\
0.039\end{array}$ & $\begin{array}{l}-0.438(-0.93 \text { to } 0.05) \\
0.081\end{array}$ & $\begin{array}{l}-0.477(-0.78 \text { to }-0.18) \\
0.002\end{array}$ \\
\hline $\begin{array}{l}\text { Fluoroscopy dose } \\
\text { (Hedges' g) }\end{array}$ & $\begin{array}{l}-0.386(-0.56 \text { to }-0.21) \\
<0.0005\end{array}$ & $\begin{array}{l}-0.279(-0.70 \text { to } 0.14) \\
0.190\end{array}$ & $\begin{array}{l}\text { Not performed, too few } \\
\text { studies }\end{array}$ & $\begin{array}{l}-0.429(-0.67 \text { to }-0.19) \\
<0.0005\end{array}$ \\
\hline
\end{tabular}

*This group contains only cryoballoon studies.

AT, atrial tachyarrhythmia; CF, contact force. 
Acute Pulmonary Vein Reconnections, PAF-only Studies

\begin{tabular}{lcccc} 
Study name & \multicolumn{3}{c}{ Statistics for each study } \\
& $\begin{array}{c}\text { Odds } \\
\text { ratio }\end{array}$ & $\begin{array}{c}\text { Lower } \\
\text { limit }\end{array}$ & $\begin{array}{c}\text { Upper } \\
\text { limit }\end{array}$ & p-Valu \\
& & & & \\
Andrade 2014 & 0.176 & 0.05 & 0.59 & 0.005 \\
Bun 2017 & 0.632 & 0.14 & 2.84 & 0.549 \\
Iwasawa 2017 & 12.803 & 0.68 & 241.03 & 0.089 \\
Marijon 2014 & 0.556 & 0.12 & 2.57 & 0.452 \\
Martinek 2012 & 0.242 & 0.06 & 1.04 & 0.057 \\
Pedrote 2016 & 0.958 & 0.06 & 16.24 & 0.976 \\
Sciarra 2014 & 0.412 & 0.12 & 1.39 & 0.153 \\
Ullah 2016 & 0.596 & 0.38 & 0.94 & 0.026 \\
& 0.486 & 0.29 & 0.82 & 0.007
\end{tabular}

Figure 5 Acute pulmonary vein reconnection, paroxysmal AF subset analysis. AF, atrial fibrillation; PAF, paroxysmal atrial fibrillation; SmartTouch, THERMOCOOL SMARTTOUCH catheter.

reported this outcome in terms of PVs, PV pairs or number of patients with acute reconnection; however, no discrepancies in effect size were found despite the variability of the outcome definition. When an alternate meta-analytic method, HKSJ, was employed, the statistical results were in agreement with the DL method (OR $0.448,95 \%$ CI 0.28 to $0.71, \mathrm{p}=0.003)$.

\section{Procedural efficiency}

Procedure time was shorter with $\mathrm{CF}$, with a moderate effect size (Hedges' g: $-0.254, \mathrm{p}=0.046$ ) (figure 6). For the subset of studies which provided procedure time means and SD, this amounted to an approximate $15.5 \mathrm{~min}$ $(9.0 \%)$ reduction. Fluoroscopy time was significantly lower in the CF group (Hedges' $g-0.442$, $\mathrm{p}<0.0005$ ). For the subset of studies which provided fluoroscopy time means and SD, this amounted to an approximate $4.8 \mathrm{~min}$ (18.7\%) reduction. Fluoroscopy time with ST trended lower compared with single-shot catheter but did not reach statistical significance (Hedges' $\mathrm{g}:-0.438, \mathrm{p}=0.081$ ) (table 2). Fewer studies reported fluoroscopy dose, but results were generally similar to those of fluoroscopy time (table 2). Procedure and fluoroscopy times varied among studies/centres, likely due to differences in procedure workflow and definition of start and stop times. Statistical results were similar when the alternate meta-analytic method (HKSJ) was employed. Study design was not a significant source of heterogeneity for the procedure and fluoroscopy time outcomes.

\section{Safety}

Twenty-four studies reported total procedural complications, of which 10 reported either no events or no major events in either CF-treated or comparator patients. Rates of complications in the remaining 14 studies ranged from $2 \%$ to $17 \%$ (table 3 ). No safety differences between ST and comparator groups were evident. The Cochran-Mantel-Haenszel $\chi^{2}$ test found no significant difference in the rate of total complications between the two groups $(\mathrm{p}=0.143, \mathrm{n}=2454)$ with a Mantel-Haenszel OR of 0.714 (95\% CI 0.45 to 1.12). There were no periprocedural deaths reported among patients treated with the CF catheter. One patient in the non-CF arm of a large multicentre study from the UK had an atrioesophageal fistula after the procedure, which lead to death. ${ }^{15}$ No significant difference was found in the rate of cardiac tamponade between 
the two groups $(\mathrm{p}=0.549, \mathrm{n}=2777$; Mantel-Haenszel OR $0.782,95 \%$ CI 0.35 to 1.73 ).

Total procedural complications, and where applicable characterisation of major or minor events, are as reported by study authors. Ten studies did not report the total number of procedural complications.

\section{DISCUSSION}

Based on a comprehensive literature review and prospectively planned analyses, the meta-analysis showed that ablation using an open-irrigated, spring sensor CF catheter had greater odds of long-term success in AF patients compared with other ablation catheters, most evidently in the paroxysmal AF population (OR 1.56, $\mathrm{p}=0.015$ ) without compromising safety as there were no differences in rates of periprocedural complication or cardiac tamponade. No difference was found in persistent AF patients (OR 1.287, $\mathrm{p}=0.385$ ), but only three studies were available within our literature search period suggesting further comparative studies in this population are needed. A randomised controlled trial in persistent $\mathrm{AF}$, which was published since our search cut-off date, also showed no difference between the CF-sensing ST catheter and the same catheter with $\mathrm{CF}$ blinded to the operator $(60 \%$ vs $63 \%$ freedom from AT at 12 months). ${ }^{16}$ The authors suggest that a study incorporating CF tagging or stability modules may result in better clinical outcomes, but would need to be further tested.

Four randomised controlled trials reported the difference in effectiveness for paroxysmal AF as the primary endpoint. These studies compared the ST catheter with and without the operators blinded to CF. This meta-analysis suggests that blinding of $\mathrm{CF}$ to experienced CF operators may not allow for a useful comparison as these operators have learnt proper use and manoeuvrability of the $\mathrm{CF}$ catheters, even in the absence of $\mathrm{CF}$ information. This training effect has been suggested by other groups as a potential explanation to CF-blinded comparative studies. ${ }^{16}$ In addition, in these four randomised trials, all $\mathrm{PV}$ reconnection/gaps were re-ablated to ensure that the

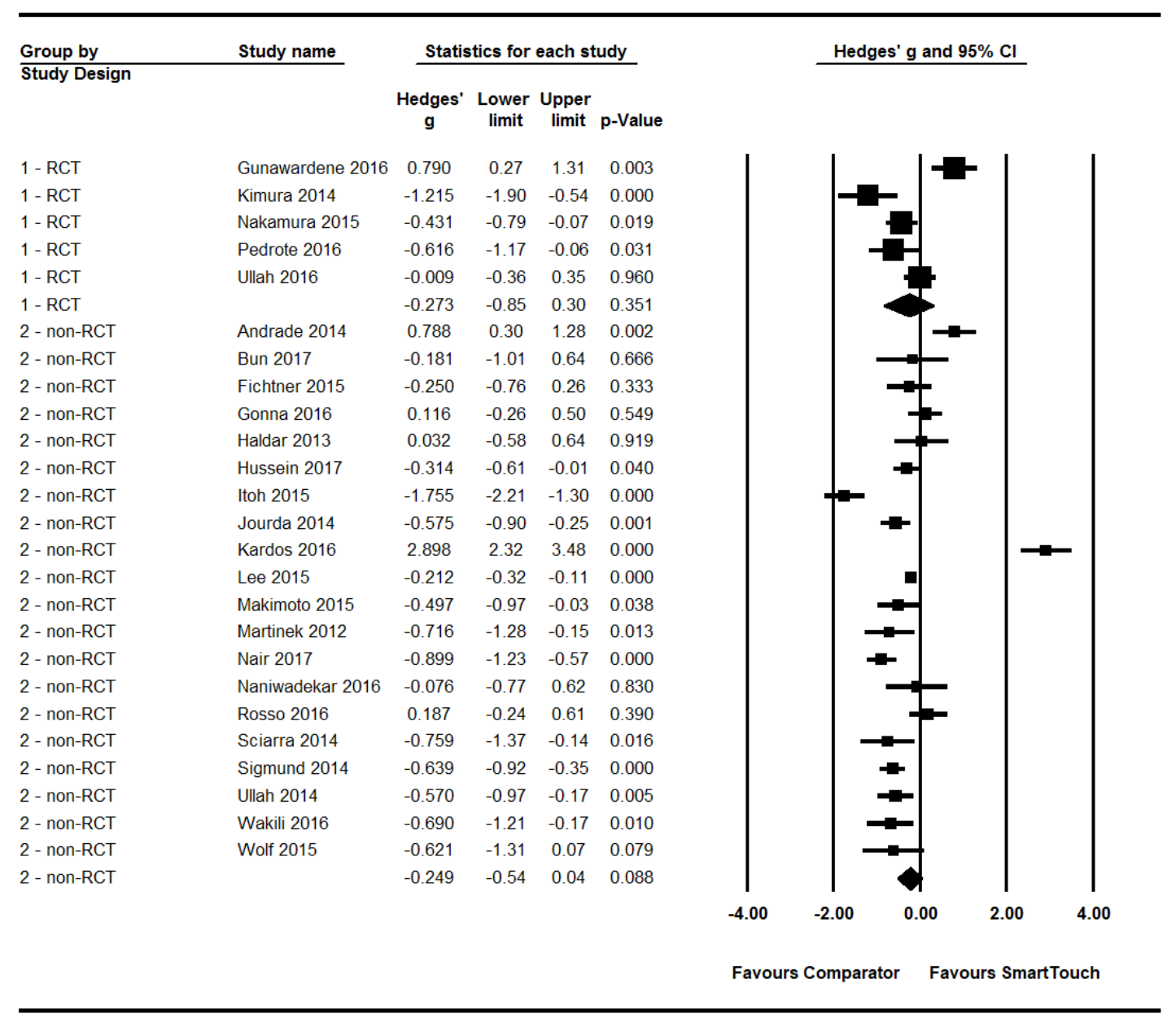

Hedges' g - Random Effects

Figure 6 Procedure time, by study design. Total effect - ST versus comparator ablation catheters: Hedges' g -0.254 , $p=0.046$. Hedges' $g$ is the measure of effect as standardised mean difference; Hedges' $g$ values $<0.2$ indicate a small effect, 0.5 a medium effect and $>0.8$ a large effect. ${ }^{9}$ RCT, randomised controlled trial; SmartTouch, THERMOCOOL SMARTTOUCH $®$ catheter. 
Table 3 Safety

\section{\# Patients with any procedural complication (Events/N)}

\begin{tabular}{llll}
\cline { 2 - 3 } Author, Year & ST & Non-CF & Comparator \\
\hline $\begin{array}{llll}\text { Fichtner et al } \\
2015^{23}\end{array}$ & $0 / 30$ & $2($ minor $) / 29$ & Irrigated RF \\
$\begin{array}{l}\text { Hussein et al } \\
2017^{24}\end{array}$ & $3 / 77$ & $4 / 97$ & $\begin{array}{l}\text { Unspecified non- } \\
\text { CF RF catheters }\end{array}$
\end{tabular}

Notes

$\begin{array}{llll}\begin{array}{l}\text { Itoh et al } \\ 2016^{25}\end{array} & 0 / 50 & 0 / 50 & \text { Irrigated RF } \\ \begin{array}{l}\text { Jarman et al } \\ 2015^{15}\end{array} & 7 / 200 & 17 / 400 & \begin{array}{l}\text { Unspecified non- } \\ \text { CF RF catheters }\end{array}\end{array}$

Two vascular access complications in comparator group

ST: one TIA, two vascular access complications

Comparator: two existing pacemaker lead dislodgement, two vascular access complications

$-$

ST: two pericardial drains, one TIA, four vascular access complications Comparator: one atrioesophageal fistula (fatal), one stroke, one PV stenosis, two phrenic palsies, five pericardial drains, seven vascular access complications

$\begin{array}{llll}\begin{array}{l}\text { Marai et al } \\ 2016^{26}\end{array} & 0 \text { (major)/11 } & 0 \text { (major)/22 } & \text { Irrigated RF } \\ \begin{array}{l}\text { Marijon et al } \\ 2014^{27}\end{array} & 0 \text { (major)/30 } & 0 \text { (major)/30 } & \text { Irrigated RF }\end{array}$
$-$

ST: two pericardial effusions, treated conservatively; one vascular access complication

Comparator: one pericardial effusion, treated conservatively; two vascular access complications

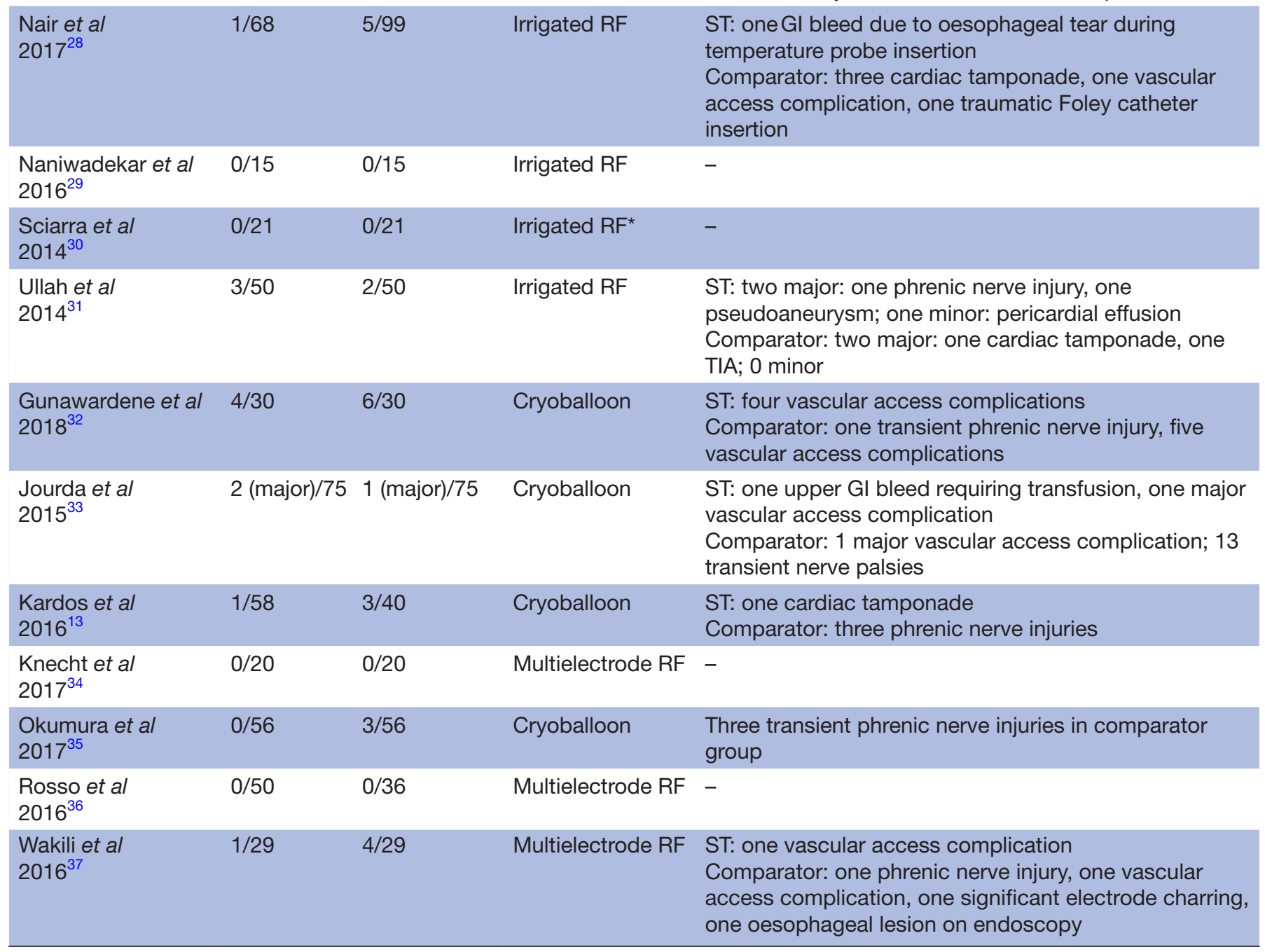


Table 3 Continued

\# Patients with any procedural complication (Events/N)

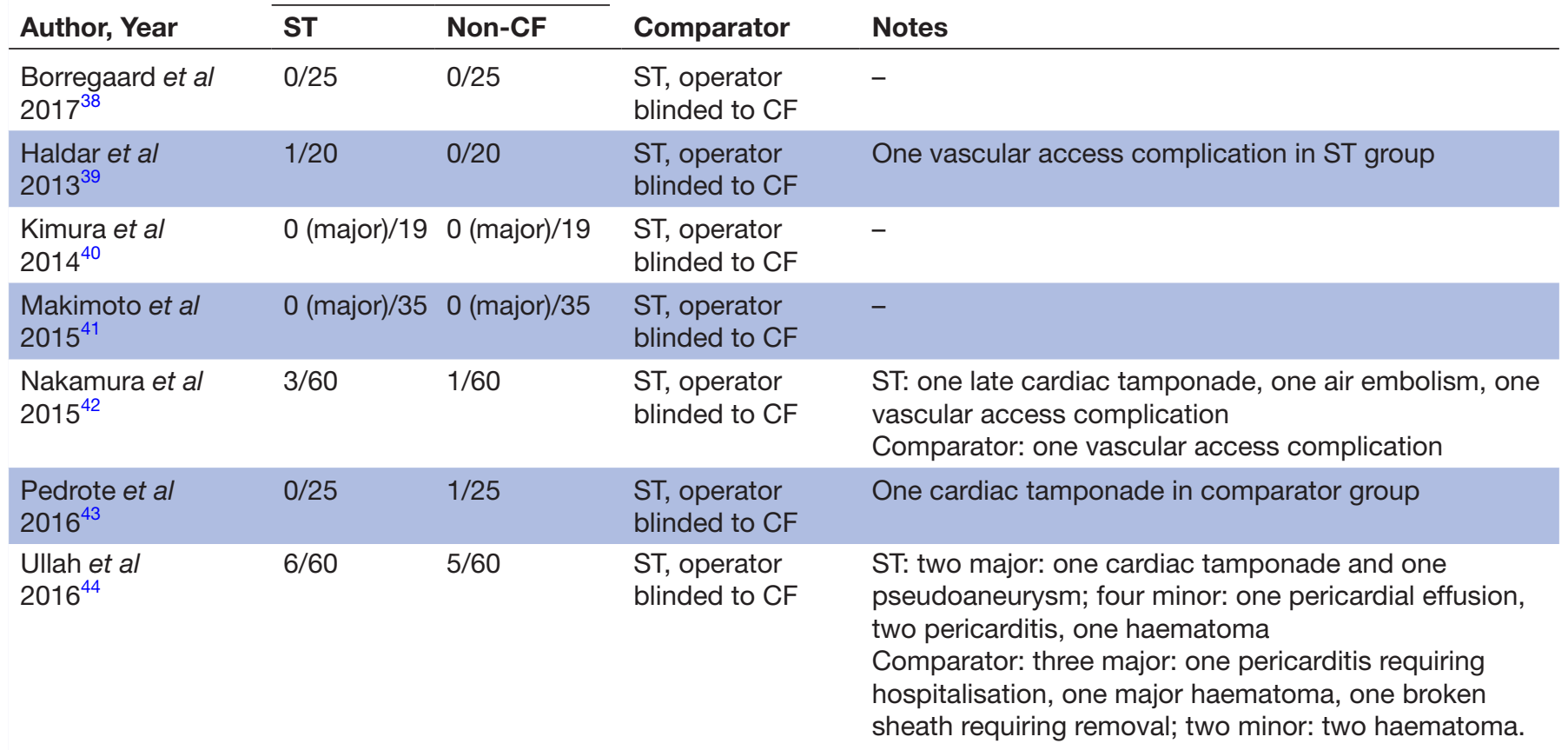

*No events in the standard Thermocool group, which was used as comparison.

$\mathrm{CF}$, contact force; Gl, gastrointestinal; N, number of patients in group; PV, pulmonary vein; RF, radiofrequency; ST, THERMOCOOL SMARTTOUCH catheter; TIA, transient ischaemic attack.

PVs were isolated, which may have minimised differences between groups. These factors will need to be considered in future randomised controlled trials.

Acute PV reconnection has been shown to be a predictor of long-term arrhythmia recurrence. ${ }^{17}$ The present meta-analysis shows that acute PV reconnection was significantly lower in the CF group, potentially explaining the differences in longer-term success given that catheter-tissue contact is critical in lesion formation. ${ }^{18}$

Moderate procedural efficiency gains were noted in the $\mathrm{CF}$ group. The moderate effect size likely reflects the variabilities of ablation workflow among different centres and early operator experience with CF catheters in these studies. A more recent study suggested a similar reduction in fluoroscopy exposure using the CF-sensing catheter. ${ }^{19}$ Most studies (9/15) in this meta-analysis did not use additional CF stability modules or automated lesion tagging software that are now available. One study showed that long-term effectiveness outcome improved with automated lesion tagging tool incorporating inter-tag distance. ${ }^{20}$ This preliminary finding will need to be confirmed in larger studies using these newer CF technologies.

This review investigated ST versus any other ablation catheter or ST with the operator blinded to CF data. In addition, safety, efficiency and efficacy were also reported with the three comparator groups (operator blinded to $\mathrm{CF}$, single-shot catheter and non-CF RF catheters) given as sub-analyses. As discussed above, there were no significant differences in freedom from AT or fluoroscopy dose when the operator was blinded from $\mathrm{CF}$ data (all within randomised controlled trials). Interestingly, although single-shot devices such as cryoballoon ablation are commonly perceived to be less time consuming than focal radiofrequency $\mathrm{PV}$ isolation, the data showed no significant difference in procedure time compared with the ST group. Fluoroscopy time trended lower in the ST group compared with single-shot device but did not reach statistical significance. Procedural efficiencies are dependent on operator experience which is related to workflow adopted by operators, including fluoroscopy used. This may have contributed to the lack of differences observed in these comparisons. As newer CF and single-shot technologies become available, it would be important to understand how workflows evolve and quantify their impacts on procedural efficiencies. No differences in effectiveness were observed between ST and single-shot devices.

The ST group showed significantly higher odds of freedom from AT at 12 months in comparison to the non-CF-sensing catheter intervention group (OR 1.766 95\% CI 1.22 to $2.55, \mathrm{p}=0.002$ ) which included point-bypoint RF catheters, a remote navigated RF catheter, a diamond-tip RF catheter and unspecified RF catheters. Procedure time, fluoroscopy time and fluoroscopy dose were also significantly lower in the ST group in comparison to non-CF catheter intervention. 
There are some noted differences between the current report and previous meta-analyses. ${ }^{45}$ Mixed CF-sensing technologies were grouped together in previous analyses. One of the analyses reported effectiveness outcomes at varying follow-up time points, ${ }^{4}$ while the other included data from abstract presentations. ${ }^{5}$ The current study employed a more focused methodology: consistent 12-month follow-up was used for the primary effectiveness outcome and only fully published results were included. Although all three meta-analyses reported that CF-guided ablation resulted in better success, the current report found the largest effect size. This may be due to differences in methodologies, incremental addition of new published evidence since the earlier reviews, greater operator experience with newer technologies in the more recent publications or possible differences in clinical outcomes between the two $\mathrm{CF}$ technologies as suggested by differences in CF relationships and clinical outcomes. ${ }^{4}$

\section{Limitations}

Limitations of this systematic review and meta-analysis primarily relate to the availability, comparability and currency of published evidence. We included data from both randomised and non-randomised studies in our analysis, due to the limited availability of randomised evidence in this setting. We are aware of two randomised trials using the ST catheter published since our search cut-off date, both of which compared use of CF-sensing ST to ST ablation with CF blinded to the operator. ${ }^{16} 21$ One of these studies, in the setting of persistent AF, is discussed above. A 2018 study conducted by Schaeffer et al randomised paroxysmal $\mathrm{AF}$ patients to two target CF settings or to operator-blinded CF; freedom from AT at 14 months was $81.9 \%$ in the high CF group and $73.5 \%$ in the lower CF group, compared with $71.4 \%$ for blinded CF ( $p=0.6$ for the comparison). ${ }^{21}$ These results were not substantially different from our meta-analysis of randomised trials overall, or the subset meta-analysis of CF-sensing ST compared with blinded CF.

Recent research on meta-analysis has provided evidence for concordance of results from randomised and non-randomised studies investigating identical outcomes. ${ }^{22}$ Moreover, we addressed potential effects of study design on the results via sensitivity and subgroup analyses. Similarly, since author definitions of the primary endpoint (freedom from AT at 12 months) and site practices for procedural parameters varied, we conducted sensitivity analyses to assess the effect of these differences. The planned sensitivity analyses found the results to be robust. Limited persistent AF studies reporting the primary endpoint were available in our analysis, and the results in this patient population need to be examined further. Procedural complications were not reported consistently from study to study, and with the small numbers of patients in most studies, it is difficult to estimate the risk of rare events.

\section{CONCLUSIONS}

Compared with ablation with other ablation catheter technologies, AF ablation using an open-irrigated catheter with spring sensor CF-sensing technology is associated with greater long-term freedom from AT and shorter procedure and fluoroscopy times.

\section{Author affiliations}

${ }^{1}$ Department of Medicine, Institut De Cardiologie de Montreal, Montreal, Quebec, Canada

${ }^{2}$ Department of Medicine, Université de Montréal, Montreal, Quebec, Canada ${ }^{3}$ Real World Evidence (RWE), CTI Clinical Trial and Consulting Services Inc., Covington, Kentucky, USA

${ }^{4}$ Cardiology, Arrhythmia and Ablation Center, Florida Hospital, Orlando, Florida, USA ${ }^{5}$ Clinical Research, Biosense Webster, Inc., Irvine, California, USA

Acknowledgements The authors thank Megan Estes, Erin Baker and Maciej Gonek for providing editorial assistance, and Leslie Pilcher for assistance with the literature review.

Contributors All authors (LM, DF, LMG, GM, SJP, LMB) contributed towards (1) conception and design or analysis and interpretation of data; (2) drafting of the manuscript and revising it critically for important intellectual content; (3) final approval of the submitted manuscript; (4) agreement to be held accountable for all aspects of the work in ensuring that questions related to the accuracy or integrity of any part of the work are appropriately investigated and resolved.

Funding This work was supported by Biosense Webster, Inc., Irvine, California, USA.

Competing interests $L M$ is a consultant to Biosense Webster, Inc., Boehringer Ingelheim, Bayer HealthCare, LLC, Servier, Medtronic, Inc., Pfizer, Inc. and St. Jude Medical and has received research grant funding from Boehringer Ingelheim, Biosense Webster, Inc. and St. Jude Medical. GM and SJP are consultants to Biosense Webster, Inc. LMG is an employee of, and DF is a consultant to, CTI Clinical Trial and Consulting Services Inc., which is a consultant to Biosense Webster, Inc. LMB is an employee of Biosense Webster, Inc. The authors retained full control of manuscript content development, including revision.

Patient consent for publication Not required.

Provenance and peer review Not commissioned; externally peer reviewed.

Data sharing statement The data sharing statement is not applicable as this is a meta-analysis of published literature.

Open access This is an open access article distributed in accordance with the Creative Commons Attribution Non Commercial (CC BY-NC 4.0) license, which permits others to distribute, remix, adapt, build upon this work non-commercially, and license their derivative works on different terms, provided the original work is properly cited, appropriate credit is given, any changes made indicated, and the use is non-commercial. See: http://creativecommons.org/licenses/by-nc/4.0/.

\section{REFERENCES}

1. January CT, Wann LS, Alpert JS, et al. AHA/ACC/HRS guideline for the management of patients with atrial fibrillation: a report of the American College of Cardiology/American Heart Association Task Force on Practice Guidelines and the Heart Rhythm Society. J Am Coll Cardiol 2014;2014:e1-76.

2. Nair GM, Raut R, Bami K, et al. Efficacy of adjunctive measures used to assist pulmonary vein isolation for atrial fibrillation: a systematic review. Curr Opin Cardiol 2017;32:58-68.

3. Reddy VY, Pollak S, Lindsay BD, et al. Relationship between catheter stability and 12-month success after pulmonary vein isolation: a subanalysis of the SMART-AF Trial. JACC Clin Electrophysiol 2016;2:691-9.

4. Shurrab M, Di Biase L, Briceno DF, et al. Impact of contact force technology on atrial fibrillation ablation: a meta-analysis. J Am Heart Assoc 2015;4:e002476.

5. Afzal MR, Chatta J, Samanta A, et al. Use of contact force sensing technology during radiofrequency ablation reduces recurrence of atrial fibrillation: A systematic review and meta-analysis. Heart Rhythm 2015;12:1990-6. 
6. Natale A, Reddy VY, Monir G, et al. Paroxysmal AF catheter ablation with a contact force sensing catheter: results of the prospective, multicenter SMART-AF trial. J Am Coll Cardiol 2014;64:647-56.

7. Reddy VY, Shah D, Kautzner J, et al. The relationship between contact force and clinical outcome during radiofrequency catheter ablation of atrial fibrillation in the TOCCATA study. Heart Rhythm 2012;9:1789-95.

8. Liberati A, Altman DG, Tetzlaff J, et al. The PRISMA statement for reporting systematic reviews and meta-analyses of studies that evaluate health care interventions: explanation and elaboration. PLoS Med 2009;6:e1000100.

9. Cohen J. The statistical power of abnormal-social psychological research: a review. J Abnorm Soc Psychol 1962;65:145-53.

10. IntHout J, loannidis JP, Borm GF. The Hartung-Knapp-Sidik-Jonkman method for random effects meta-analysis is straightforward and considerably outperforms the standard DerSimonian-Laird method. BMC Med Res Methodol 2014;14:25.

11. Rücker G, Schwarzer G, Carpenter J. Arcsine test for publication bias in meta-analyses with binary outcomes. Stat Med 2008;27:746-63.

12. In: Higgins JPT, Green S, eds. Cochrane handbook for systematic reviews of interventions. Version 5.1.0: The Cochrane Collaboration, 2011.

13. Kardos A, Kis Z, Som Z, et al. Two-year follow-up after contact force sensing radiofrequency catheter and second-generation cryoballoon ablation for paroxysmal atrial fibrillation: a comparative single centre study. Biomed Res Int 2016;2016:1-6.

14. Nagy Z, Kis Z, Som Z, et al. [Catheter ablation for paroxysmal atrial fibrillation: new generation cryoballoon or contact force sensing radiofrequency ablation?]. Orv Hetil 2016;157:849-54.

15. Jarman JWE, Panikker S, Das M, et al. Relationship between contact force sensing technology and medium-term outcome of atrial fibrillation ablation: a multicenter study of 600 patients. $J$ Cardiovasc Electrophysiol 2015;26:378-84.

16. Conti S, Weerasooriya R, Novak P, et al. Contact force sensing for ablation of persistent atrial fibrillation: A randomized, multicenter trial. Heart Rhythm 2018;15:201-8.

17. Anter E, Contreras-Valdes FM, Shvilkin A, et al. Acute pulmonary vein reconnection is a predictor of atrial fibrillation recurrence following pulmonary vein isolation. $J$ Interv Card Electrophysiol 2014;39:225-32.

18. Avitall B, Mughal K, Hare J, et al. The effects of electrodetissue contact on radiofrequency lesion generation. Pacing Clin Electrophysiol 1997;20:2899-910

19. Voskoboinik A, Kalman ES, Savicky $Y$, et al. Reduction in radiation dose for atrial fibrillation ablation over time: A 12-year single-center experience of 2344 patients. Heart Rhythm 2017;14:810-6.

20. Phlips T, Taghji P, El Haddad M, et al. Improving procedural and oneyear outcome after contact force-guided pulmonary vein isolation: the role of interlesion distance, ablation index, and contact force variability in the 'CLOSE'-protocol. Europace 2018;20:f419-f427.

21. Schaeffer B, Willems S, Meyer C, et al. Contact force facilitates the achievement of an unexcitable ablation line during pulmonary vein isolation. Clin Res Cardiol 2018;107:632-41.

22. Anglemyer A, Horvath HT, Bero L. Healthcare outcomes assessed with observational study designs compared with those assessed in randomized trials. Cochrane Database Syst Rev 2014:MR000034.

23. Fichtner S, Reents T, Ammar S, et al. Pulmonary vein isolation using new technologies to improve ablation lesion formation: Initial results comparing enhanced catheter tip irrigation (Surround Flow(®)) with contact force measurement (Smarttouch(®))). Indian Pacing Electrophysiol J 2015;15:152-7.

24. Hussein A, Das M, Chaturvedi V, et al. Prospective use of ablation index targets improves clinical outcomes following ablation for atrial fibrillation. J Cardiovasc Electrophysiol 2017;28:1037-47.

25. Itoh $\mathrm{T}$, Kimura M, Tomita $\mathrm{H}$, et al. Reduced residual conduction gaps and favourable outcome in contact force-guided circumferential pulmonary vein isolation. Europace 2016;18:531-7.

26. Marai I, Suleiman M, Blich M, et al. Impact of computed tomography image and contact force technology on catheter ablation for atrial fibrillation. World J Cardiol 2016;8:317-22.

27. Marijon E, Fazaa S, Narayanan K, et al. Real-time contact force sensing for pulmonary vein isolation in the setting of paroxysmal atrial fibrillation: procedural and 1-year results. $J$ Cardiovasc Electrophysiol 2014;25:130-7.

28. Nair GM, Yeo C, MacDonald Z, et al. Three-year outcomes and reconnection patterns after initial contact force guided pulmonary vein isolation for paroxysmal atrial fibrillation. $J$ Cardiovasc Electrophysiol 2017;28:984-93

29. Naniwadekar A, Joshi K, Greenspan A, et al. Use of the new contact force sensing ablation catheter dramatically reduces fluoroscopy time during atrial fibrillation ablation procedures. Indian Pacing Electrophysiol J 2016;16:83-7.

30. Sciarra L, Golia P, Natalizia A, et al. Which is the best catheter to perform atrial fibrillation ablation? A comparison between standard ThermoCool, SmartTouch, and Surround Flow catheters. J Interv Card Electrophysiol 2014;39:193-200.

31. Ullah W, Hunter RJ, Haldar S, et al. Comparison of robotic and manual persistent AF ablation using catheter contact force sensing: an international multicenter registry study. Pacing Clin Electrophysiol 2014;37:1427-35.

32. Gunawardene MA, Hoffmann BA, Schaeffer B, et al. Influence of energy source on early atrial fibrillation recurrences: a comparison of cryoballoon vs. radiofrequency current energy ablation with the endpoint of unexcitability in pulmonary vein isolation. Europace 2018;20:euw307.

33. Jourda F, Providencia R, Marijon E, et al. Contact-force guided radiofrequency vs. second-generation balloon cryotherapy for pulmonary vein isolation in patients with paroxysmal atrial fibrillation-a prospective evaluation. Europace 2015;17:225-31.

34. Knecht S, Sticherling C, Reichlin T, et al. Reliability of luminal oesophageal temperature monitoring during radiofrequency ablation of atrial fibrillation: insights from probe visualization and oesophageal reconstruction using magnetic resonance imaging. Europace 2017;19:euw129.

35. Okumura Y, Watanabe I, Iso $\mathrm{K}$, et al. Mechanistic insights into durable pulmonary vein isolation achieved by second-generation cryoballoon ablation. J Atr Fibrillation 2017;9:1538.

36. Rosso R, Chorin E, Levi Y, et al. Radiofrequency ablation of atrial fibrillation: nonrandomized comparison of circular versus pointby-point "smart" ablation for achieving circumferential pulmonary vein isolation and curing arrhythmic symptoms. J Cardiovasc Electrophysiol 2016;27:1282-7.

37. Wakili R, Siebermair J, Fichtner S, et al. One-year clinical outcome after ablation with a novel multipolar irrigated ablation catheter for treatment of atrial fibrillation: potential implications for clinical use. Europace 2016;18:1170-8.

38. Borregaard R, Jensen HK, Tofig BJ, et al. Is the knowledge of contact force beneficial in pulmonary vein antrum isolation? Scand Cardiovasc J 2017:51:129-37.

39. Haldar S, Jarman JW, Panikker S, et al. Contact force sensing technology identifies sites of inadequate contact and reduces acute pulmonary vein reconnection: a prospective case control study. Int $J$ Cardiol 2013;168:1160-6.

40. Kimura M, Sasaki S, Owada S, et al. Comparison of lesion formation between contact force-guided and non-guided circumferential pulmonary vein isolation: a prospective, randomized study. Heart Rhythm 2014;11:984-91.

41. Makimoto $\mathrm{H}$, Heeger $\mathrm{CH}$, Lin T, et al. Comparison of contact force-guided procedure with non-contact force-guided procedure during left atrial mapping and pulmonary vein isolation: impact of contact force on recurrence of atrial fibrillation. Clin Res Cardiol 2015;104:861-70.

42. Nakamura K, Naito S, Sasaki T, et al. Randomized comparison of contact force-guided versus conventional circumferential pulmonary vein isolation of atrial fibrillation: prevalence, characteristics, and predictors of electrical reconnections and clinical outcomes. J Interv Card Electrophysiol 2015;44:235-45.

43. Pedrote A, Arana-Rueda E, Arce-León A, et al. Impact of contact force monitoring in acute pulmonary vein isolation using an anatomic approach. A randomized study. Pacing Clin Electrophysiol 2016;39:361-9.

44. Ullah W, McLean A, Tayebjee MH, et al. Randomized trial comparing pulmonary vein isolation using the SmartTouch catheter with or without real-time contact force data. Heart Rhythm 2016;13:1761-7. 